\title{
The Hyperfine Coupling Radical Pair Mechanism of Biological Effects on Weak Magnetic Fields
}

\author{
Jin-Hua Ouyang ${ }^{1}$ \\ ${ }^{\prime}$ College of Physics and Engineering, Qufu Normal University, Qufu, China \\ Email:yjhua@163.com Tel:86-13805474172
}

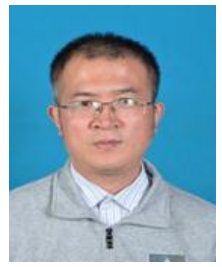

\section{Abstract}

More recent concerns have been raised about the biological effects of all different weak magnetic fields (MFs) with the development of electric device era and information era. The biological effects due to weak MFs just like a hypomagnetic field (HMF) or near-null magnetic field is a very important subject for aerospace traveling and space station living. A large number of studies on the biological effects of other weak MFs have been carried out. The biological effects of different weak MFs can be negative or positive. Many mechanisms to explain the bioeffects of weak MFs have been given. Are there possible common mechanisms of the different biological effects in different weak MFs ? It's unbelievable and sound impossible at first sight that different bioeffects of different weak MFs have common mechanisms. But possible common mechanisms can exist. In this study, the author proposes the relations between the singlet yield of the radical pair and the weak MFs are the possible common mechanisms. Giving one possible common mechanism of bioeffects of all different weak MFs including impulsed ones is the first to the author's known. Here, the weak MFs are ones that cannot produce thermal bioeffects not only limited below $1 \mathrm{mT}$.

Keywords: Hyperfine coupling radical pair mechanism, Weak magnetic fields, Biological effects, Hypomagnetic field (HMF), Near-null magnetic field, and Singlet yield of the radical pair.

Citation | Jin-Hua Ouyang (2019). The Hyperfine Coupling Radical Pair Mechanism of Biological Effects on Weak Magnetic Fields. Asian Engineering Review, 6(1): 1-8

History:

Received: 31 December 2018

Revised: 24 January 2019

Accepted: 27 February 2019

Published: 29 March 2019

Licensed: This work is licensed under a Creative Common Attribution 3.0 License (oc)

Publisher: Asian Online Journal Publishing Group
Contribution/Acknowledgement: The author is sincerely grateful to Dr Wei-Chuan Mo (State Key Laboratory of Brain and Cognitive Science, Institute of Biophysics, Chinese Academy of Sciences, Beijing, China.), as well as to Dr. Bao-Ming Xu (Shandong Key Laboratory of biophysics, Dezhou University, Dezhou, China) and Dr. Hong-zhen Li (School of Physics, Qu Normal University, Qufu, China ) for their helpful discusions on thiss, Qu Funding: This work is supported by the Scientific Research Starting Foundation of Qufu Normal University (Grant No. 107/1024; 107/6019). Competing Interests: The author declares that there are no conflicts of interests regarding the publication of this paper.

Transparency: The author confirms that the manuscript is an honest, accurate, and transparent account of the study was reported; that no vital features of the study have been omitted; and that any discrepancies from the study as planned have been explained.

Ethical: This study follows all ethical practices during writing.

\section{Contents}

1. Introduction

2. General Radical Pair Model and the Hyperfine Coupling Radical Pair Mechanism in Bird Navigation

3. The Hyperfine Coupling Radical Pair Mechanism of Biological Effects on Weak MFs

4. Discussion

5. Summary

References 


\section{Introduction}

Since at least the late 1950 s, the public have raised the concerns about the potential biological effects of nonionizing radiation [1]. According to the European Commission, non-ionizing radiation are divided into static magnetic fields (o Hz), extremely low-frequency (o to $300 \mathrm{~Hz}$ ), intermediate frequency $(300 \mathrm{~Hz}$ to $100 \mathrm{kHz}$ ), and radio frequency $(100 \mathrm{kHz}$ to $300 \mathrm{GHz})$ fields [2].

The static magnetic fields (SMFs) are classified as following four grades:(1) the weak grade $(<1 \mathrm{mT}) ;(2)$ the moderate grade (1 mT -1T); (3) the strong grade (1T-5T); and (4) the ultrastrong grade $(>5 \mathrm{~T})$ [3].

Untill now a lot of biological effects of weak magnetic fields have been found and studied [4]. During the evolution process, all living organisms experienced the action of the Earth's field(geomagnetic field, GMF) which is a natural component of their environment [5]. While, Interplanetary space is a natural hypomagnetic field(HMF) [5]. The range of a HMF is below $5 \mathrm{uT}[5]$. Hypomagnetic field also can be called null or near-null magnetic field [6]. A hypomagnetic field(HMF) or near-null magnetic field is a very important subject for aerospace traveling and space station living [5]. Because of the difference on intensity between $\mathrm{GMF}(-5 \mathrm{OuT})$ and a HMF $(<5 \mathrm{uT})[5]$ many effects appear when animals, plants and microorganisms are in the HMF [5-16]. For example, a significant increase in anxiety-related behaviors was found when adult male C57BL/6 mice was in short-term $\mathrm{HMF}$ for $72 \mathrm{~h}$ [8]. Usually, GMF and HMF are considered as part of static magnetic fields (SMFs). Because of their ranges of intensity, they are the entire weak grade. The biological effects of other SMFs (except for GMF and a HMF)are reported [17-20]. More recent concerns have arisen about the potential effects of all different weak magnetic fields with the development of electric device era and information era [1]. For example, more and more people have been attracted by the small increases in childhood leukemia for children living near power lines and possible increases in brain tumors for heavy use of cell phones [1]. The biological effects of extremely low-frequency (o to $300 \mathrm{~Hz}$ ) magnetic fields (ELF-MFs) are presented [21-34]. The biological effects of intermediate frequency (30O Hz to 100 $\mathrm{kHz}$ ) magnetic fields(IMF-MFs)are discussed [35-38]. The biological effects of radio frequency (100 $\mathrm{kHz}$ to 300 $\mathrm{GHz}$ ) magnetic fields (RF-MFs) are talked about [39-42]. The biological effects of pulsed magnetic fields are reported [43-47]. The biological effects of weak MFs can also be positive. For example ,a single session of Pulsed magnetic fields (parameters: $0.5 \mathrm{mT}, 12 \mathrm{~Hz}$, and $30 \mathrm{~min}$ ) produced significantly greater increase in peripheral blood flow velocity in the dorsal foot [43] which may be used to treat some peripheral diseases.

Many mechanisms to explain the bioeffects of weak MFs have been given. Electromagnetic induction,ferrimagnetism and radical pair mechanisms of biological effects of a GMF are introduced [48-54]. Radical pair and magnetic moment mechanisms of biological effects of a HMF are suggested [7]; [55-57]. The mechanisms of biological effects of ELF-MFs which are cyclotron resonance theory and it's new interpretation,the ion parametric resonance model and an interference of ions bound within proteins are proposed [58-61]. The radical pair mechanism of biological effects of RF-MFs are outlined [1]; [62, 63]. Because magnetic fields in the band of $300 \mathrm{~Hz}$ through $10 \mathrm{MHz}$ between ELF-MFs and RF-MFs have not been sufficiently explored so far [64] the mechanisms of biological effects of an intermediate frequency magnetic fields(IMF-MFs) have not been reported to the author's known. From the mechanisms of different biological effects of different magnetic fields, one can see the mechanisms are different too. Is there one common mechanism among the different biological effects of different weak magnetic fields? The study gives one possible answer. The hyperfine coupling radical pair mechanism in bird navigation is demonstrated [65]. The author thinks that the hyperfine coupling radical pair mechanism in bird navigation can be extended to explain biological effects of other weak magnetic fields. That is to say the hyperfine coupling radical mechanism or the relations between the singlet yield of the radical pair and the weak MFs may be common ones among the different mechanisms to explain different biological effects of different weak magnetic fields. In this study, the weak MFs are ones that cannot produce thermal bioeffects not only limited below $1 \mathrm{mT}$. The reason is given in part 4.

The presentation order of the contents of this paper is as follows. General radical pair model and the hyperfine coupling radical pair mechanism in bird navigation is shown in part 2; the hyperfine coupling radical pair mechanism of biological effects on weak MFs is presented in part 3; Part 4 is discussions of several questions on the hyperfine coupling radical pair mechanism of weak MFs; and the main contents are summarized in part 5.

\section{General Radical Pair Model and the Hyperfine Coupling Radical Pair Mechanism in Bird Navigation}

A radical can be a molecule that contains an odd number of electrons. A radical pair consists of two radicals that have been created simultaneously, usually by a chemical reaction [66]. The reaction scheme can be divided into three steps [67]. The first one is that an excited donor molecule transfers an electron to an acceptor molecule resulting in a radical pair. Once the radical pair is generated, its singlet and triplet states will be interconverted by the hyperfine interaction. The final one is that singlet and triplet pairs will react to give singlet or triplet products $[67,68]$.

The hyperfine coupling radical model in bird navigation is given as followed [65]. When considering that the vertical hyperfine coupling $A_{z}$ is relatively strong compared with $\mathcal{B}_{0}$, and the horizontal hyperfine coupling $A_{x}=A_{y}=0$ is assumed, the singlet yield

$$
\phi_{S}^{0}(\theta) \approx \frac{1}{2}-\frac{1}{4} \sin ^{2} \theta-\frac{\gamma^{2} B_{0}^{2}}{A_{Z}^{2}}\left(\frac{3}{4} \sin ^{2} \theta-\sin ^{4} \theta\right),
$$

can be obtained under the influence of the geomagnetic field with the corresponding eigenvalue,here,which is equal to $\gamma B_{0}$, where $B_{0}$ is the intensity of the Earth's magnetic field, and $\theta$ describes its orientation to the basis of the hyperfine tensor and $\gamma$ is one constant [65]. When considering the horizontal hyperfine coupling $A_{x}=A_{y} \neq 0$ ,the results are similar to that without considering it $\mathrm{Xu}$, et al. [65]. [65]

When considering the influence of an additional weak oscillating field, the singlet yield is given in Xu, et al. 


$$
\begin{aligned}
& \qquad \phi_{S} \approx \begin{cases}\phi_{S}^{0}(\theta) & \omega \neq 2 \omega_{0} \\
\phi_{S}^{0}(\theta)+\frac{1}{2}\left(\phi_{+}^{\prime}(\theta)+\phi_{-}^{\prime}(\theta)\right) & \omega=2 \omega_{0}\end{cases} \\
& \text { with } \\
& \qquad \phi_{ \pm}^{\prime}(\theta) \approx-\frac{1}{4} \frac{\gamma^{2} B_{r f}^{2}}{k^{2}} \sin ^{2} \varphi \cos ^{2}\left(\theta_{ \pm}-\theta\right), \omega_{0}=\gamma B_{0} .
\end{aligned}
$$

Where, $1 / k$ is the radical pair lifetime. $\theta_{ \pm}$is determined by the surrounding magnetic field of electron and the hyperfine coupling $A_{z}$, and $B_{r f}$ is the strength of additional oscillating field with frequency $\omega$.From Equation 3, it can be seen that if the radio frequency field is parallel to the geomagnetic field, i.e., $\varphi=0, \phi_{ \pm}^{\prime}(\theta)=0$, it means that the parallel radio frequency field has no influence on the singlet yield. If the radio frequency field is not parallel to the geomagnetic field, i.e., $\varphi \neq 0$, the radio frequency field influences the singlet yield [65].

\section{The Hyperfine Coupling Radical Pair Mechanism of Biological Effects on Weak MFs}

\subsection{The Hyperfine Coupling Mechanism of Biological Effects on GMF}

Magnetic sensing to detect the Earth's magnetic field, is still one of the most controversial animal senses because the exact molecules to sense GMF have not been found [51]. Some models have been proposed to solve the controversy [51]. The author thinks the primary physical mechanism of the ability to detect the Earth's magnetic field is Equation 1 or 2 just as the same as the bird navigation in part 2. From Equation 2 and Equation 3 one can see when the time-dependent field is parallel to the geomagnetic field, i.e., $\varphi=0, \phi_{ \pm}^{\prime}(\theta)=0$, or it's frequency is not equal to two folds of the corresponding eigenvalue,the biological effects are caused only by the geomagnetic (static)field. Only when the time-dependent field is not parallel to the geomagnetic field, i.e., $\varphi \neq 0$, and it's frequency is equal to two folds of the corresponding eigenvalue,the biological effects are caused by both it and the geomagnetic (static)field.Here, the time-dependent field can either be external one made by human beings or one of the ambient alternating fields, because the wireless electromagnetic noise frequency of the surroundings can be below $1 \mathrm{~Hz}[69]$ from $1 \mathrm{~Hz}$ to $100 \mathrm{KHz}[70]$ from $9 \mathrm{KHz}$ to $10 \mathrm{GHz}$ and above $10 \mathrm{GHz}[71]$ and sometimes, the noise can be very important [72].

\subsection{The Hyperfine Coupling Mechanism of Biological Effects on a HMF}

Because of the difference on intensity between $\operatorname{GMF}(\sim 50 \mathrm{TT})$ and a $\operatorname{HMF}(<5 \mathrm{uT})[5]$ many effects appear when animals, plants and microorganisms are in the HMF [5-16]. For example, a HMF can significantly suppress Arabidopsis flowering in light [14]. Because of the $\mathrm{HMF}$ as a key environmental factor when long-term and longdistance space mission in outer space, and also in some magnetic shielding conditions on the ground,the biological effect of the HMF should be seriously considered [73]. Some studies on the mechanisms underlying the biological effects of a HMF have been carried out [6]. These studies give the mechanism of the biological effects of a HMF at the cellular level [74-80] the mechanism at the molecular level [11]; [47]; [81-88] the mechanism at the tissue level $[89,90]$ and the physical mechanism respectively [55-57]. However, the underlying mechanisms remain unclear. In Ouyang and $\mathrm{Li}[7]$ the author and his partner gave the point: Equation 1 may be one quantum mechanism of biological effects on a HMF. Ambient alternating fields always exist $[73,74]$ can they influence the biological effects on a HMF ? The author thinks ambient alternating fields can influence the biological effects on a HMF just like on GMF as discussed in part 2.That is to say Equation 2 may be one possible quantum mechanism of the biological effects of a HMF when considering ambient alternating fields. In Zhang, et al. [73] the $\operatorname{HMF}\left(\boldsymbol{B}_{0}\right)$ is $0.19 \pm 0.08(u T)$ or $0.14 \pm 0.07(u T)$. According to $\omega_{0}=\gamma B_{0}, \omega=2 \omega_{0}$, the frequency of ambient alternating fields that can influence the biological effects is from $3076(\mathrm{~Hz})$ to $7550(\mathrm{~Hz})$ or from $1958(\mathrm{~Hz})$ to $5872(\mathrm{~Hz})$. In Zhang, et al. [73] the ambient alternating fields is $2200-2700(\mathrm{~Hz})$ or $2300-2900(\mathrm{~Hz})$. Based on Equation 2, together with common range between $1958-5872(\mathrm{~Hz})$ and $2300-2900(\mathrm{~Hz})$, the biological effects are results of a HMF combined with ambient alternating fields. That is to say, ambient alternating fields can influence the biological effects of a HMF when the double eigenvalue $\omega_{0}=\gamma B_{0}, \omega=2 \omega_{0}$ is included in the frequency of ambient alternating fields. Choleris, et al. [91] also gave the point that ambient alternating fields can influence the biological effects of a HMF. To the contrast if the double eigenvalue $\omega_{0}=\gamma B_{0}, \omega=2 \omega_{0}$ is not included in the frequency of ambient alternating fields, the ambient alternating fields can not influence the biological effects of a HMF. Because Equation 2 is obtained based on the time-dependent perturbation theory [65] when ambient alternating fields is not much weaker compared with $B_{0}$, it can not be used.

\subsection{The Hyperfine Coupling Mechanism of Biological Effects on Other SMFS (Except for GMF and A HMF)}

Weak static magnetic field (500uT) increased total lipid content, including polar lipids, among them glycolipids and phospholipids was given in Novitskii, et al. [17]. Tenuzzo, et al. [92] results found apoptosis increased in some cells and decreased in the other when the cells were exposed in $6 \mathrm{mT}$ static magnetic fields. Wang, et al. [93] results showed that $1.00 \mathrm{~T}$ and $1.13 \mathrm{~T}$ SMFs could increase cellular adenosine triphosphate (ATP). The biological effects of other SMFs(except for GMF and a HMF) were shown in Novitskii, et al. [17]; Sullivan, et al. [18]; Tolosa, et al. [19]; Martino [20].

The wireless electromagnetic noise frequency of the surroundings can be below $1 \mathrm{~Hz}[69]$ from $1 \mathrm{~Hz}$ to $100 \mathrm{KHz}[70]$ from $9 \mathrm{KHz}$ to $10 \mathrm{GHz}$ and above $10 \mathrm{GHz}[71]$. Sometimes, the noise can be very important $[72]$. 
Based on $\omega_{0}=\gamma B_{0}, \omega=2 \omega_{0}$, when $B_{0}$ equals $0.01 \mathrm{mT}, \omega=2 \omega_{0}$ is about $300 \mathrm{KHz}$; when $B_{0}$ equals $1 \mathrm{mT}$, $\omega=2 \omega_{0}$ is about $30 \mathrm{MHz}$; when $B_{0}$ equals $100 \mathrm{mT}, \omega=2 \omega_{0}$ is about $3 \mathrm{GHz}$; when $B_{0}$ equals $10 \mathrm{~T}, \omega=2 \omega_{0}$ is about $300 \mathrm{GHz}$.

Because of the frequency richness of the wireless electromagnetic noise of the surroundings and the weakness of it's intensity,usually the double eigenvalue $\omega_{0}=\gamma B_{0}, \omega=2 \omega_{0}$ is included in the frequency of ambient alternating fields and the ambient alternating fields is much weaker compared with $B_{0}$.

From these that can be seen, the biological effects of other SMFs(except for a HMF and GMF) can be explained by Equation 2. That is to say,the biological effects of other SMFs(except for GMF and a HMF) are caused by both the wireless electromagnetic noise of the surroundings and the SMFs usually. Here, because of no external alternating field, the wireless electromagnetic noise of the surroundings is the alternating field, $B_{0}$ is the sum both GMF and SMFs when GMF and SMFs have the same directions. When they have different directions, $B_{0}$ is smaller than the sum both GMF and SMFs.

\subsection{The Hyperfine Coupling Mechanism of Biological Effects on ELF-MFs}

Because of industrialization, characterized by an increasing production, distribution, and consumption of electricity, occupational exposure to ELF-MFs in the frequency range of $3 \mathrm{~Hz}$ to $300 \mathrm{~Hz}$ is common and many workers are occupationally exposed to these fields Jalilian, et al. [94]. Bawin, et al. [95]; Bawin and Adey [96] provided important initial findings of non-thermal ELF-MFs that $\mathrm{Ca}^{2+}$ efflux from chick embryo brain tissue was influenced by ELF-MFs.Importantly, Blackman, et al. [97] later confirmed these results. An increased risk of amyotrophic lateral sclerosis (ALS) in workers occupationally exposed to ELF-MFs were observed in Huss, et al. [21]. Kleijn, et al. [22]; Zhang, et al. [23]; Schüz, et al. [24]; Tang, et al. [25]; Fixler, et al. [26]; Wang, et al. [27]; Prato, et al. [28]; Corbacio, et al. [29]; Vanderstraeten and Gillis [30]; Bowman, et al. [31]; Prato, et al. [32]; Burda, et al. [33]; Burger, et al. [34] reported the other biological effects of ELF-MFs (o to 30O Hz) fields. Smith, et al. [58]; Lednev [59]; Blanchard and Blackman [60]; Binhi [61] proposed the mechanisms of biological effects of ELF-MFs which are cyclotron resonance theory and it's new interpretation,the ion parametric resonance model and an interference of ions bound within proteins. These mechanisms can not explain the role of light in the course of biological effects appearing [82]. The radical pair mechanism is valid for static MFs, but is also applicable to power frequency MFs: for radical recombination times, which are in the order of tens of nanosecond to few microseconds, $50 / 60 \mathrm{~Hz}$ fields may be regarded as static [98]. Based on this, the author thinks almost ELFMFs effects can be explained through Equation 2.

Here, $B_{0}$ is the sum both GMF and amplitude of ELF-MFs. Just like the case of GMF or HMF, the author thinks ambient alternating fields can influence the biological effects on ELF-MFs. That is to say, ambient alternating fields can influence the biological effects of ELF-MFs when the double eigenvalue $\omega_{0}=\gamma B_{0}, \omega=2 \omega_{0}$ is included in the frequency of ambient alternating fields.

To the contrast, if the double eigenvalue $\omega_{0}=\gamma B_{0}, \omega=2 \omega_{0}$ is not included in the frequency of ambient alternating fields, the ambient alternating fields can not influence the biological effects of ELF-MFs. Because Equation 2 is obtained based on the time-dependent perturbation theory [65] when ambient alternating fields is not much weaker compared with $B_{0}$, it can not be used just like the case of GMF or a HMF.

\subsection{The Hyperfine Coupling Mechanism of Biological Effects on an IMF-MFs}

The intermediate frequency magnetic fields in the context of protecting human exposure have received public concerns in recent years because of the growing emergence of technology or products using those fields and a number of studies have been carried out Yamazaki, et al. [64]. Kumari, et al. [35] findings suggest that exposure to a $7.5 \mathrm{kHz}, 120 \mu \mathrm{T}$ MF may lead to mild learning and memory impairment possibly through an inflammatory reaction in the hippocampus. From Navarro, et al. [37] results, it seems that a low intensity $2 \mathrm{kHz}$ exposure modifies short-term working memory, as well as perception, binary decision, motor execution, and sustained attention. Barbault, et al. [38]; Capstick, et al. [39] reported that cancer cell growth may be altered by very low level magnetic fields modulated at specific frequencies in humans.

Because IMF-MFs is $300 \mathrm{~Hz}$ through $100 \mathrm{KHz}$ or $10 \mathrm{MHz}$ [2]; [64] ELF-MFs is oHz through $300 \mathrm{~Hz}$ and the author thinks ELF-MFs may be regarded as static and corresponding effects can be explained through Equation 2 in part 3.4, and one band of IMF-MFs (near ELF-Mfs) may be regarded as static and can be explained through Equation 2 just like the case of ELF-MFs.

When the frequency is so larger that IMF-MFs may not be regarded as static, how corresponding effects can be explained? This problem can be conquered together with RF-MFs.

\subsection{The Hyperfine Coupling Mechanism of Biological Effects on RF-MFs}

With the development of the globally advancing digitalization, the use of mobile communication systems and the mobile devices is more and more, which results in a rise of RF-MFs exposure of the human body Köhler, et al. [99]. Valentini, et al. [100] concluded that mobile phone RF-MFs may influence normal physiology. Apollonio, et al. [101] reported the other biological effects of RF-MFs. For a long time, there have been substantial concerns over the possible effects of RF-MFs at exposure levels that do not lead to significant increases in temperature [62]. Possible health effects of RF-MFs on the human body are still in discussion and have not finally been verified $[100$, 101]. Thermal effect which leads to temperature increase in cells and tissue and is based on dielectric heating is the undisputed and possibly only interaction of RF-MFs with biological cells and tissue [99]; [101]. However, the occurrence of non-thermal effects within cells, tissue and organism are still one controversial discussion [63]; [99101]. Nevertheless, one mechanism that explains the observed effects with certainty is not known [40-42]. 
Based on $\omega_{0}=\gamma B_{0}, \omega=2 \omega_{0}$, when $B_{0}$ equals $0.01 \mathrm{mT}, \omega=2 \omega_{0}$ is about $300 \mathrm{KHz}$; when $B_{0}$ equals $1 \mathrm{mT}$, $\omega=2 \omega_{0}$ is about $30 \mathrm{MHz}$; when $B_{0}$ equals $100 \mathrm{mT}, \omega=2 \omega_{0}$ is about $3 \mathrm{GHz}$; when $B_{0}$ equals $10 \mathrm{~T}, \omega=2 \omega_{0}$ is about $300 \mathrm{GHz}$. The wireless electromagnetic noise frequency of the surroundings can be below $1 \mathrm{~Hz}$ [69] from $1 \mathrm{~Hz}$ to $100 \mathrm{KHz}[70]$ from $9 \mathrm{KHz}$ to $10 \mathrm{GHz}$ and above $10 \mathrm{GHz}[71]$. Sometimes, the noise can be very important [72].

From these the author thinks, the biological effects of an IMF-MFs or a RF-MFs can be explained by Equation 2.

Here, because of IMF-MFs or RF-MFs as external field, the wireless electromagnetic noise of the surroundings and external field all can be the alternating field, and $B_{0}$ is the sum both GMF and amplitude of low frequency part of the wireless electromagnetic noise that may be regarded as static just like the case of part 3.3.

\subsection{The Hyperfine Coupling Radical Pair Mechanism of Biological Effects on Pulsed Magnetic Fields}

Chen, et al. [44] results suggested that early an impulsed electromagnetic field therapy produced early and significant benefit in central nervous regeneration. Sun, et al. [43]; Hei, et al. [45]; Zhai, et al. [46]; Martino, et al. [47] reported other biological effects of an impulsed magnetic field. Because an impulse signal can be divided into different frequency parts, an impulsed MF is combined with ELF-MFs, IMF-MFs and RF-MFs, and the biological effects of an impulsed MF is comprehensive results with ELF-MFs, IMF-MFs and RF-MFs. That is to say, the biological effects of impulsed MFs can be explained by Equation 2. Here, because of impulsed MFs as external field, the wireless electromagnetic noise of the surroundings and external field all can be the alternating field, and $B_{0}$ is the sum both GMF and amplitude of low frequency part of the wireless electromagnetic noise plus the base or harmonics of the impulsed MFs that may be regarded as static. Prato [82] showed that induction of analgesia could be very significantly increased if an impulsed magnetic field was used rather than a sinuidal field. The result can be explained very well using the hyperfine coupling radial pair mechanism.Because the harmonics of the impulsed MFs satisfied the double eigenvalue $\omega_{0}=\gamma B_{0}, \omega=2 \omega_{0}$, the difference of results between a pulsed magnetic field and a sinuidal field is caused based on Equation 2.

\section{Discussion}

Some references [1]; [25]; [29]; [36]; [42]; [62]; [87]; [94]; [101]; [102] discussed the problems of the irreproducible bioeffects of weak magnetic fields. Some references $[1] ;[36] ;[62] ;[101] ;[102]$ explained the reasons in different view points. What are the primary physical mechanisms of the problems? In this study the author gives one possible common mechanism of bioeffects of weak magnetic fields. In this mechanism, the noise can be very important sometimes. Because of the differences of the noise in different time and different place, some bioeffects experimental results on weak magnetic fields may be not same.

In the different bioeffects experiments of different weak magnetic fields, only ones of hypomagnetic(or nearnull magnetic) fields are good in reproduction to the author's knowledge. Why is this? It is one aim of next research. In part $2, B_{r f}$ is much weaker compared with $B_{0}$ is assumed, so the time-dependent perturbation theory can be used to calculate the yield of the singlet state. When $B_{r f}$ is not much weaker compared with $B_{0}$, that is to say the noise is not very much weak compared with external magnetic fields, what is the influence of the noise accordingly? It is the the other aim of next research.

In part 2, $A_{z}$ is assumed relatively strong compared with the external field in Xu, et al. [65] Equation 2 is given. When $A_{z}$ is not relatively strong compared with the external field, Equation 2 is not the mechanism of bioeffects of weak magnetic fields.

In part 1,the arthor gives that in this study, the weak MFs are ones that can not produce thermal bioeffects not only limited below 1mT. Why? Though [67] expressed that for strong magnetic fields (>100mT) singlet-triplet interconversion can occur by virtue of the different Zeeman interactions, that is to say,the hyperfine coupling radical pair mechanism may have weaker influence in biological effects compared to the magnetic fields below $1 \mathrm{mT}$, Hore and Mouritsen [66] pointed out that tiny interactions can have profound effects but only if the system has previously been brought into an appropriate state. The author thinks the weaker influence of the hyperfine coupling radical pair mechanism may be the tiny interaction when the magnetic fields is stronger than $1 \mathrm{mT}$.

When the static magnetic field is stronger than $200 \mathrm{mT}$, water can be magnetized [103]. Magnetized water maybe have some magnetic bioeffects along with above hyperfine coupling radical pair mechanisms in organisms. When the magnetic field is so strong that it can produce thermal bioeffects, the bioeffects produced through above hyperfine coupling radical pair mechanisms may be mixed with thermal ones.

\section{Summary}

The primary physical mechanism which can be related to the biological effects of weak magnetic fields is one key problem and has not been clarified. In this article,the author builds one bridge that has connected the bird navigation and bioeffects of other magnetic fields in possible common explanatory mechanisms, because the author expands Equations 1 and 2 to be used to other weak magnetic fields including impulsed ones. That is to say the author proposes one hyperfine coupling radical pair mechanism in all weak magnetic fields and explains the reasons of the irreproducible bioeffects of them. Giving one possible common mechanism of bioeffects of all different weak MFs including an impulsed ones is the first to the author's known. Here, the weak MFs are ones that can not produce thermal bioeffects not only limited below $1 \mathrm{mT}$. 


\section{References}

[1] F. Barnes and B. Greenenbaum, "Some effects of weak magnetic fields on biological systems: RF fields can change radical concentrations and cancer cell growth rates," IEEE Power Electronics Magazine, vol. 3, pp. 60-68, $2016 . \quad$ Available at: https://doi.org/10.1109/mpel.2015.2508699.

[2] M. M.-Z. Khoshroo, M. S. Mehrjan, F. Samiee, M. Soltani, and S. P. H. Shekarabi, "Some immunological responses of common carp (Cyprinus Carpio) fingerling to acute extremely low-frequency electromagnetic fields (50 Hz)," Fish Physiology and Biochemistry, vol. 44, pp. 235-243, 2018. Available at: https://doi.org/10.1007/s10695-017-0429-1.

[3] S. Ghodbane, A. Lahbib, M. Sakly, and H. Abdelmelek, "Bioeffects of static magnetic fields: Oxidative stress, genotoxic effects, and cancer studies," BioMed Research International, vol. 2013, pp. 1-12, 2013. Available at: https://doi.org/10.1155/2013/602987.

[4] U. E. Steiner and T. Ulrich, "Magnetic field effects in chemical kinetics and related phenomena," Chemical Revierws, vol. 89, pp. 51-147, 1989. Available at: https:// doi.org/10.1021/crooo91a003

[5] W. Mo, Y. Liu, and R. He, "Hypomagnetic field, an ignorable environmental factor in space," Science China Life Sciences, vol. 57, pp. 726728, 2014. Available at: https://doi.org/10.1007/s11427-014-4662-x.

[6] W. C. Mo, Y. Liu, and R. Q. He, "A biological perspective of the hypomagnetic field: From definition towards mechanism," Progress in Biochemistry and Biophysics, vol. 39, pp. 835-842, 2012. Available at: 10.3724/SP.J.1206.2011.00597.

[7] J. H. Ouyang and H. Z. Li, "Biological effects due to hypomagnetic field and its research progress," Journal of Life Sciences Research, vol. 4, pp. 14-19, 2017. Available at: https://doi.org/10.20448/journal.504.2017.42.14.19.

H. M. Ding, X. Wang, W. C. Mo, L. L. Qin, S. Wong, J. P. Fu, Y. Tan, Y. Liu, R. Q. He, and Q. Hua, "Hypomagnetic fields cause anxietyin adult male mice," Bioelectromagnetics, vol. 40, pp. 27-32, 2019. Available at: https://doi.org/10.1002/bem.22155.

[9] W. C. Mo, Z. J. Zhang, Y. Liu, G. J. Zhai, Y. D. Jiang, and R. Q. He, "Effects of a hypogeomagnetic field on gravitropism and germination in soybean," Advances in Space Research, vol. 47, pp. 1616-162 1, 2011. Available at: https://doi.org/10.1016/j.asr.2010.12.024.

[10] G. J. Wan, S. L. Jiang, Z. C. Zhao, J. J. Xu, X. R. Tao, G. A. Sword, Y. B. Gao, W. D. Pan, and F. J. Chen, "Bio-effects of near-zero magnetic fields on the growth, development and reproduction of small brown planthopper, laodelphax striatellus and brown planthopper, ilaparvata lugens," Journal of Insect Physiology, vol. 68, pp. 7-15, 2014. Available at: https://doi.org/10.1016/j.jinsphys.2014.06.016.

[11] C. Xu, X. Yin, Y. Lv, C. Wu, Y. Zhang, and T. Song, "A near-null magnetic field affects cryptochrome-related hypocotyl growth and flowering in arabidopsis," Advances in Space Research, vol. 49, pp. 834-840, 2012. Available at: https://doi.org/10.1016/j.asr.2011.12.004.

[12] C. Xu, S. Wei, Y. Lu, Y. Zhang, C. Chen, and T. Song, "Removal of the local geomagnetic field affects reproductive growth in arabidopsis," Bioelectromagnetics, vol. 36, pp. 437-442, 2013. Available at: https://doi.org/10.1002/bem.21788.

[13] C. Xu, Y. Lv, C. Chen, Y. Zhang, and S. Wei, "Blue light-dependent phosphorylations of cryptochromes are affected by magnetic fields in Arabidopsis," Advances in Space Research, vol. 53, pp. 1118-1124, 2014. Available at: https://doi.org/10.1016/j.asr.2014.01.033.

[14] C. Xu, Y. Li, Y. Yu, Y. Zhang, and S. Wei, "Suppression of arabidopsis flowering by near-null magnetic field is affected by light," Bioelectromagnetics, vol. 36, pp. 476-479, 2015. Available at: https://doi.org/10.1002/bem.21927

[15] W. C. Mo, Y. Liu, H. M. Cooper, and R. Q. He, "Altered development of Xenopus embryos in a hypogeomagnetic field," Bioelectromagnetics, vol. 33, pp. 238-246, 2012. Available at: https://doi.org/10.1002/bem.20699.

[16] W. C. Mo, J. P. Fu, H. M. Ding, Y. Liu, Q. Hua, and R. Q. He, "Hypomagnetic field alters circadian rhythm and increases algesia in adult male mice," Progress in Biochemistry and Biophysics, vol. 42, pp. 639-646, 2015. Available at: 10.16476/j.pibb.2015.0137.

[17] Y. Novitskii, G. Novitskaya, and Y. Serdyukov, "Effect of weak permanent magnetic field on lipid composition and content in perilla leaves," Bioelectromagnetics, vol. 37, pp. 108-115, 2016. Available at: https://doi.org/10.1002/bem.21957.

[18] K. Sullivan, A. K. Balin, and R. G. Allen, "Effects of static magnetic fields on the growth of various types of human cells," Bioelectromagnetics, vol. 32, pp. 140-147, 2011. Available at: https://doi.org/10.1002/bem.20624.

[19] M. F. Tolosa, C. Bouzat, and W. R. Cravero, "Effects of static magnetic fields on nicotinic cholinergic receptor function," Bioelectromagnetics, vol. 32, pp. 434-442, 2011. Available at: https://doi.org/10.1002/bem.20657.

[20] C. F. Martino, "Static magnetic field sensitivity of endothelial cells," Bioelectromagnetics, vol. 32, pp. 506-508, 2011. Available at: https://doi.org/10.1002/bem.20665.

[21] A. Huss, S. Peters, and R. Vermeulen, "Occupational exposure to extremely low-frequency magnetic fields and the risk of ALS: A systematic review and meta-analysis," Bioelectromagnetics, vol. 39, pp. 156-163, 2018. Available at: https://doi.org/10.1002/bem.22104.

[22] S. Kleijn, G. Ferwerda, M. Wiese, J. Trentelman, J. Cuppen, T. Kozicz, v. Verburg, and B. L. Kemenade, "A short-term extremely low frequency electromagnetic field exposure increases circulating leukocyte numbers and affects HPA-axis signaling in mice," Bioelectromagnetics, vol. 37, pp. 433-443, 2016. Available at: https://doi.org/10.1002/bem.21998.

[23] J. Zhang, C. Xu, Y. Wan, and M. Gao, "Effects of extremely low frequency magnetic field on production of mannatide by $\alpha$-hemolytic streptococcus," Bioelectromagnetics, vol. 37, pp. 331-337, 2016. Available at: https://doi.org/10.1002/bem.21984.

[24] J. Schüz, C. Dasenbrock, P. Ravazzani, M. Röösli, P. Schär, P. L. Bounds, and Y. Hamnerius, "Extremely low-frequency magnetic fields and risk of childhood leukemia: A risk assessment by the ARIMMORA consortium," Bioelectromagnetics, vol. 37, pp. 183-189, 2016. Available at: https://doi.org/10.1002/bem.21963.

[25] R. Tang, Y. Xu, F. Ma, J. Ren, S. Shen, Y. Du, and T. Wang, "Extremely low frequency magnetic fields regulate differentiation of regulatory T cells: Potential role for ROS-mediated inhibition on AKT," Bioelectromagnetics, vol. 37, pp. 89-98, 2016. Available at: https://doi.org/10.1002/bem.21954.

[26] D. Fixler, S. Yitzhaki, A. Axelrod, T. Zinman, and A. Shainberg, "Correlation of magnetic AC field on cardiac myocyte Ca2+ transients at different magnetic DC levels," Bioelectromagnetics, vol. 33, pp. 634-640, 2012. Available at: https://doi.org/10.1002/bem.21729.

[27] T. Wang, Y. Nie, S. Zhao, Y. Han, Y. Du, and Y. Hou, "Involvement of midkine expression in the inhibitory effects of low-frequency magnetic fields on cancer cells," Bioelectromagnetics, vol. 32, pp. 443-452, 2011. Available at: 10.1002/bem.20654.

[28] F. S. Prato, D. Desjardins-Holmes, L. D. Keenliside, J. M. DeMoor, J. A. Robertson, R. Z. Stodilka, and A. W. Thomas, "The detection threshold for extremely low frequency magnetic fields may be below $1000 \mathrm{nT}-\mathrm{Hz}$ in mice," Bioelectromagnetics, vol. 32, pp. 561-569, 2011. Available at: 10.1002/bem.20661.

[29] M. Corbacio, S. Brown, S. Dubois, D. Goulet, F. S. Prato, A. W. Thomas, and A. Legros, "Human cognitive performance in a 3 mT powerline frequency magnetic field," Bioelectromagnetics, vol. 32, pp. 620-633, 2011. Available at: https://doi.org/10.1002/bem.20676.

[30] J. Vanderstraeten and P. Gillis, "Theoretical evaluation of magnetoreception of power-frequency fields," Bioelectromagnetics, vol. 31 , pp. 371-379, 2010. Available at: https://doi.org/10.1002/bem.20568.

[31] J. D. Bowman, C. K. Miller, E. F. Krieg, and R. Song, "Analyzing digital vector waveforms of 0-3000 Hz magnetic fields for health studies " Bioelectromagnetics, vol. 31, pp. 391-405, 2010. Available at: https://doi.org/10.1002/bem.20570.

[32] F. S. Prato, D. Desjardins-Holmes, L. D. Keenliside, J. M. DeMoor, J. A. Robertson, and A. W. Thomas, "Magnetoreception in laboratory mice: Sensitivity to extremely low-frequency fields exceeds $33 \mathrm{nT}$ at $30 \mathrm{~Hz}, "$ Journal of the Royal Society Interface, vol. 10, pp. 2012104620121046, 2013. Available at: https://doi.org/10.1098/rsif.2012.1046.

[33] H. Burda, S. Begall, J. Červený, J. Neef, and P. Němec, "Extremely low-frequency electromagnetic fields disrupt magnetic alignment of ruminants," Proceedings of the National Academy of Sciences, vol. 106, pp. 5708-5713, 2009. Available at: https://doi.org/10.1073/pnas.0811194106.

[34] T. Burger, M. Lucová, R. E. Moritz, H. H. Oelschläger, R. Druga, H. Burda, W. Wiltschko, R. Wiltschko, and P. Němec, "Changing and shielded magnetic fields suppress c-Fos expression in the navigation circuit: Input from the magnetosensory system contributes to the internal representation of space in a subterranean rodent," Journal of The Royal Society Interface, vol. 7, pp. 1275-1292, 2010. Available at: https://doi.org/10.1098/rsif.2009.0551.

[35] K. Kumari, H. Koivisto, M. Viluksela, K. M. Paldanius, M. Marttinen, M. Hiltunen, J. Naarala, H. Tanila, and J. Juutilainen, "Behavioral testing of mice exposed to intermediate frequency magnetic fields indicates mild memory impairment," PLoS One, vol. 12, pp. e0188880e0188880, 2017. Available at: https://doi.org/10.1371/journal.pone.0188880

[36] T. Shigemitsu, K. Yamazaki, S. Nakasono, and M. Kakikawa, "A review of studies of the biological effects of electromagnetic fields in the intermediate frequency range," IEE Journal of Transactions, vol. 2, pp. 405-412, 2007. Available at: https://doi.org/10.1002/tee.20181. E. A. Navarro, C. Gomez-Perretta, and F. Montes, "Low intensity magnetic field influences short-term memory: A study in a group of healthy students," Bioelectromagnetics, vol. 37, pp. 37-48, 2016. Available at: https://doi.org/10.1002/bem.21944.

[38] A. Barbault, F. P. Costa, B. Bottger, R. F. Munden, F. Bomholt, N. Kuster, and B. Pasche, "Amplitude-modulated electromagnetic fields for the treatment of cancer: Discovery of tumor-specific frequencies and assessment of a novel therapeutic approach," Journal of Experimental E Clinical Cancer Research, vol. 28, pp. 1-10, 2009. Available at: https://doi.org/10.1186/1756-9966-28-51. 
[39] M. Capstick, Y. Gong, B. Pasche, and N. Kuster, "An HF exposure system for mice with improved efficiency," Bioelectromagnetics, vol. 37, pp. 223-233, 2016. Available at: https://doi.org/10.1002/bem.21969.

[40] P. R. Castello, I. Hill, F. Sivo, L. Portelli, F. Barnes, R. Usselman, and C. F. Martino, "Inhibition of cellular proliferation and enhancement of hydrogen peroxide production in fibrosarcoma cell line by weak radio frequency magnetic fields," Bioelectromagnetics, vol. 35, pp. 598602, 2014. Available at: https://doi.org/10.1002/bem.21858.

[41] E. Schleicher, R. Wenzel, M. Ahmad, A. Batschauer, L.-O. Essen, K. Hitomi, E. D. Getzoff, R. Bittl, S. Weber, and A. Okafuji, "The electronic state of flavoproteins: Investigations with proton electron-nuclear double resonance," Applied Magnetic Resonance, vol. 37, pp. 339-352, 2010. Available at: https://doi.org/10.1007/s00723-009-0101-8.

[42] I. Calvente, R. Pérez-Lobato, M. I. Núñez, R. Ramos, M. Guxens, J. Villalba, N. Olea, and M. F. Fernández, "Does exposure to environmental radiofrequency electromagnetic fields cause cognitive and behavioral effects in 10-year-old boys?," Bioelectromagnetics, vol. 37, pp. 25-36, 2016. Available at: https://doi.org/10.1002/bem.21951.

[43] J. Sun, R. L.-C. Kwan, Y. Zheng, and G. L.-Y. Cheing, "Effects of pulsed electromagnetic fields on peripheral blood circulation in people with diabetes: A randomized controlled trial," Bioelectromagnetics, vol. 37, pp. 290-297, 2016. Available at: https://doi.org/10.1002/bem.21983.

[44] Q. Chen, G.-m. Lin, N. Wu, S.-w. Tang, Z.-j. Zheng, M. C.-m. Lin, G.-x. Xu, H. Liu, Y.-y. Deng, and X.-y. Zhang, "Early exposure of rotating magnetic fields promotes central nervous regeneration in planarian girardia sinensis," Bioelectromagnetics, vol. 37, pp. 244-255, 2016. Available at: https://doi.org/10.1002/bem.21971

[45] W. H. Hei, S. Kim, J. C. Park, Y. K. Seo, S. M. Kim, J. W. Jahng, and J. H. Lee, "Schwann-like cells differentiated from human dental pulp stem cells combined with a pulsed electromagnetic field can improve peripheral nerve regeneration," Bioelectromagnetics, vol. 37, pp. 163174, 2016. Available at: https://doi.org/10.1002/bem.21966.

[46] M. Zhai, D. Jing, S. Tong, Y. Wu, P. Wang, Z. Zeng, G. Shen, X. Wang, Q. Xu, and E. Luo, "Pulsed electromagnetic fields promote in vitro osteoblastogenesis through a Wnt/ $\beta$-catenin signaling-associated mechanism," Bioelectromagnetics, vol. 37, pp. 152-162, 2016. Available at: https://doi.org/10.1002/bem.21961.

[47] C. F. Martino, H. Perea, U. Hopfner, V. L. Ferguson, and E. Wintermantel, "Effects of weak static magnetic fields on endothelial cells," Bioelectromagnetics, vol. 31, pp. 296-301, 2010. Available at: https://doi.org/10.1002/bem.20565.

[48] S. Johnsen and K. J. Lohmann, "Magnetoreception in animals," Physics Today, vol. 61, pp. 29-35, 2008. Available at: https://doi.org/10.1063/1.2897947.

[49] S. Zhan, C. Merlin, J. L. Boore, and S. M. Reppert, "The monarch butterfly genome yields insights into long-distance migration," Cell, vol. 147, pp. 1171-1185, 2011. Available at: https://doi.org/10.1016/j.cell.2011.09.052.

[50] W. Wiltschko and R. Wiltschko, "Magnetic orientation and magnetoreception in birds and other animals," Journal of Comparative Physiology A, vol. 191, pp. 675-693, 2005. Available at: https://doi.org/10.1007/s00359-005-0627-7.

[51] S. Y. Qin, H. Yin, C. L. Yang, Y. F. Dou, Z. Liu, P. Zhang, H. Yu, Y. L. Huang, J. Feng, J. F. Hao, J. Hao, L. Z. Deng, X. Y. Yan, X. L. Dong, Z. X. Zhao, T. Jiang, H. W. Wang, S. J. Luo, and C. Xie, "A magnetic protein biocompass," Nature Materials, vol. 15, pp. 217-226, 2016. Available at: https://doi.org/10.1038/nmat4484.

[52] R. C. Beason, "Mechanisms of magnetic orientation in birds," Integrative and Comparative Biology, vol. 45, pp. 565-573, 2005. Available at: https://doi.org/10.1093/icb/45.3.565.

[53] M. M. Walker and M. E. Bitterman, "Conditioning analysis of magnetoreception in honeybees," Bioelectromagnetics, vol. 10, pp. 261-275, 1989. Available at: https://doi.org/10.1002/bem.2250100305.

[54] M. Winklhofer, E. Dylda, P. Thalau, W. Wiltschko, and R. Wiltschko, "Avian magnetic compass can be tuned to anoma-lously low magnetic intensities," Proceedings of the Royal Society B, vol. 280, pp. 20130853-20130853, 2013. Available at: https://doi.org/10.1098/rspb.2013.0853.

[55] V. Binhi, "Primary physical mechanism of the biological effects of weak magnetic fields," Biophysics, vol. 61, pp. 170-176, 2016. Available at: https://doi.org/10.1134/s000635091601005x.

[56] V. N. Binhi and F. S. Prato, "A physical mechanism of magnetoreception: Extension and analysis," Bioelectromagnetics, vol. 38, pp. 41-52, 2017. Available at: https://doi.org/10.1002/bem.22011.

[57] V. Binhi and F. Prato, "Biological effects of the hypomagnetic field: An analytical review of experiments and theories," PLoS One, vol. 12, pp. e0179340-e0 179340, 2017. Available at: https://doi.org/10.1371/journal.pone.0179340.

[58] S. D. Smith, B. R. McLeod, A. R. Liboff, and K. Cooksey, "Calcium cyclotron resonance and diatom mobility," Bioelectromagnetics, vol. 8, pp. 215-227, 1987. Available at: https://doi.org/10.1002/bem.2250080302.

[59] V. Lednev, "Possible mechanism for the influence of weak magnetic fields on biological systems," Bioelectromagnetics, vol. 12, pp. 71-75, 1991. Available at: https://doi.org/10.1002/bem.2250120202.

[60] J. P. Blanchard and C. F. Blackman, "Clarification and application of an ion parametric resonance model for magnetic field interactions with biological systems," Bioelectromagnetics, vol. 15, pp. 2 17-238, 1994. Available at: https://doi.org/10.1002/bem.2250150306.

[61] V. N. Binhi, "Amplitudeand frequency dissociation spectraof ion-protein complexes rotating in magnetic fields," Bioelectromagnetics, vol. 21 , pp. 34-45, 2000. Available at: https://doi.org/10.1002/(sici)1521-186x(200001)2 1:1<34::aid-bem6>3.0.co;2-8.

[62] F. Barnes and B. Greenebaum, "Role of radical pairs and feedback in weak radio frequency field effects on biological systems," Environmental Research, vol. 163, pp. 165-170, 2018. Available at: https://doi.org/10.1016/j.envres.2018.01.038.

[63] F. S. Barnes and B. Greenebaum, "The effects of weak magnetic fields on radical pairs," Bioelectromagnetics, vol. 36, pp. 45-54, 2015. Available at: https://doi.org/10.1002/bem.21883.

[64] K. Yamazaki, M. Taki, and C. Ohkubo, "Safety assessment of human exposure to intermediate frequency electromagnetic fields," Electrical Engineering in Japan, vol. 197, pp. 3-11, 2016. Available at: https://doi.org/10.1002/eej.22884.

[65] B.-M. Xu, J. Zou, H. Li, J.-G. Li, and B. Shao, "Effect of radio frequency fields on the radical pair magnetoreception model," Physical Review E, vol. 90, pp. 042711-042711, 2014. Available at: https://doi.org/10.1103/physreve.90.042711.

[66] P. J. Hore and H. Mouritsen, "The radical-pair mechanism of magnetoreception," Annual Review of Biophysics, vol. 45, pp. 299-344, 2016. Available at: https://doi.org/10.1146/annurev-biophys-032116-094545.

[67] T. Ritz, S. Adem, and K. Schulen, "A model for photoreceptor-based magnetoreception in birds," Biophysical Journal, vol. 78, pp. 707-718, 2000. Available at: https://doi.org/10.1016/s0006-3495(00)76629-x.

[68] K. Kominis, "Quantum Zeno effect explains magnetic-sensitive radical-ion-pair reactions," Physical Review E, vol. 80, p. 056115, 2009. Available at: https://doi.org/10.1103/physreve.80.056115.

[69] M. N. Zhadin, "Review of russian literature on biological action of DC and low-frequency AC magnetic fields," Bioelectromagnetics, vol. 22, pp. 27-45, 2001. Available at: https://doi.org/10.1002/1521-186x(200101)22:1<27::aid-bem4>3.0.co;2-2.

[70] J. J. Lu, D. L. Liu, and H. Xie, "Characteristics analysis of low frequency radio wave communication channel atmospheric noise," presented at the 2011 AASRI Conference on Artificial Intelligence and Industry Application (AASRI-AIIA 2011). Available: http://kns.cnki.net/, 2011

[71] F. Yu, C. Y. Lv, and Z. Luo, "The study of measurement of electromagnetic nosie," Information \& Communications, vol. 156, pp. 4-7, 2015.

$[72] \quad$ L. Deying, "Analysis of frequency harmonics influence on operation of EMU trains," RSCE, vol. 15, pp. 73-77, 2018. Available at: 10.3969/j.issn.1673-4440.2018.04.016.

[73] H.-t. Zhang, Z.-j. Zhang, W.-c. Mo, P.-d. Hu, H.-m. Ding, Y. Liu, Q. Hua, and R.-q. He, "Shielding of the geomagnetic field reduces hydrogen peroxide production in human neuroblastoma cell and inhibits the activity of CuZn superoxide dismutase," Protein \& Cell, vol. 8 , pp. 527-537, 2017. Available at: https://doi.org/10.1007/s13238-017-0403-9.

[74] J.-P. Fu, W.-C. Mo, Y. Liu, and R.-Q. He, "Decline of cell viability and mitochondrial activity in mouse skeletal muscle cell in a hypomagnetic field," Bioelectromagnetics, vol. 37, pp. 212-222, 2016. Available at: https://doi.org/10.1002/bem.21968.

[75] B. Jia, W.-J. Zhang, L. Xie, Q. Zheng, Z.-C. Tian, A.-R. Qian, and P. Shang, "Effects of hypomagnetic field environment on hematopoietic system in mice," Hangtian Yixue yu Yixue Gongcheng, vol. 24, pp. 318-322, 2011. Available at: 10.16289/j.cnki.1002-0837.2011.05.001.

[76] H. Ding, W. Mo, J. Fu, J. Hu, Y. Liu, and Q. Hua, "The hematopoietic system responses to onemonth continuous hypomagnetic field exposure in adult mice," Prog Mod Biomed, vol. 26, pp. 5001-5004, 2014. Available at: 10.13241/j.cnki.pmb.2014.26.001.

[77] W. Mo, Z. Zhang, Y. Liu, P. Bartlett, and R. He, "Magnetic shielding accelerates the proliferation of human neuroblastoma cell by promoting G1-phase progression," PLoS One, vol. 8, pp. e54775-e54775, 2013. Available at: https://doi.org/10.1371/journal.pone.0054775.

[78] B. Jia, L. Xie, Q. Zheng, P. Yang, W. Zhang, C. Ding, A. Qian, and P. Shang, "A hypomagnetic field aggravates bone loss induced by hindlimb unloading in rat femurs," PLoS One, vol. 9, pp. e105604-e105604, 2014. Available at: https://doi.org/10.1371/journal.pone.0105604. 
[79] J. Zhang, C. Ding, and P. Shang, "Alterations of mineral elements in osteoblast during differentiation under hypo, moderate and high static magnetic fields," Biological Trace Element Research, vol. 162, pp. 153-157, 2014. Available at: https://doi.org/10.1007/s12011-014-0157-7.

[80] W. C. Mo, Z. J. Zhang, D. L. Wang, Y. Liu, P. F. Bartlett, and R. Q. He, "Shielding of the geomagnetic field alters action assembly and inhibits cell motility in human neuroblastoma cells," Scientific Reports, vol. 6, pp. 1-15, 2016. Available at: https://doi.org/10.1038/srep22624.

[81] W. Mo, Y. Liu, P. F. Bartlett, and R. He, "Transcriptome profile of human neuroblastoma cells in the hypomagnetic field," Science China Life Sciences, vol. 57, pp. 448-46 1, 2014. Available at: https://doi.org/10.1007/s1 1427-014-4644-Z.

[82] F. S. Prato, "Non-thermal extremely low frequency magnetic field effects on opioid related behaviors: Snails to humans, mechanisms to therapy," Bioelectromagnetics, vol. 36, pp. 333-348, 2015. Available at: https://doi.org/10.1002/bem.21918.

[83] L. Foley, R. Gegear, and S. Reppert, "Human cryptochrome exhibits light-dependent magnetosensitivity," Nature Communications, vol. 2, pp. 356-356, 2011. Available at: https://doi.org/10.1038/ncomms 1364.

[84] K. Maeda, A. J. Robinson, K. B. Henbest, H. J. Hogben, T. Biskup, M. Ahmad, E. Schleicher, S. Weber, C. R. Timmel, and P. Hore, K. Maeda, A. J. Robinson, K. B. Henbest, H. J. Hogben, T. Biskup, M. Ahmad, E. Schleicher, S. Weber, C. R. Timmel, and P. Hore,
"Magnetically sensitive light-induced reactions in cryptochrome are consistent with its proposed role as a magnetoreceptor," Proceedings of the National Academy of Sciences, vol. 109, pp. 4774-4779, 2012. Available at: https://doi.org/10.1073/pnas.1118959109.

[85] D. L. Wang, X. S. Wang, R. Xiao, Y. Liu, and R. Q. He, "Tubulin assembly is disordered in a hypogeomagnetic field," Biochemical and Biophysical Research Communications, vol. 376, pp. 363-368, 2008. Available at: https://doi.org/10.1016/j.bbrc.2008.08.156.

[86] C. F. Martino, L. Portelli, K. McCabe, M. Hernandez, and F. Barnes, "Reduction of the earth's magnetic field inhibits growth rates of model cancer cell lines," Bioelectromagnetics, vol. 31, pp. 649-655, 2010. Available at: https://doi.org/10.1002/bem.20606.

[87] I. Y. Belyaev, Y. D. Alipov, and M. Harms-Ringdahl, "Effects of zero magnetic field on the conformation of chromatin in human cells," Biochimica et Biophysica Acta (BBA)-General Subjects, vol. 1336, pp. 465-473, 1997. Available at: https://doi.org/10.1016/s03044165(97)00059-7.

[88] C. F. Martino and P. R. Castello, "Modulation of hydrogen peroxide production in cellular systems by low level magnetic fields," PLoS One, vol. 6, p. e22753, 2011 . Available at: https://doi.org/10.1371/journal.pone.0022753.

[89] C. Ding, B. Jia, X. Liu, Y. Lv, J. Wang, and P. Shang, "Effects of hypo-magnetic field and simulated microgravity on dielectric properties of gastrocnemius in rats," Space Medicine \& Medical Engineering, vol. 27, pp. 1-5, 2014. Available at: 10.16289/j.cnki.1002-0837.2014.01.011.

[90] C. Ding, B. Jia, X. Liu, Y. Lv, J. Wang, and P. Shang, "Dielectric properties of rat whole blood, spleen and testis were changed by hypomagnetic field and hind-limb unloading," Space Medicine ES Medical Engineering, vol. 27, pp. 157-163, 2014. Available at: 10.16289/j.cnki.1002-0837.2014.03.008.

[91] E. Choleris, C. Del Seppia, A. Thomas, P. Luschi, S. Ghione, G. Moran, and F. Prato, "Shielding, but not zeroing of the ambient magnetic field reduces stress-induced analgesia in mice," Proceedings of the Royal Society of London. Series B: Biological Sciences, vol. 269, pp. 193-201, 2002. Available at: https://doi.org/10.1098/rspb.2001.1866.

[92] B. Tenuzzo, A. Chionna, E. Panzarini, R. Lanubile, P. Tarantino, B. D. Jeso, M. Dwikat, and L. Dini, "Biological effects of 6 mT static magnetic fields: A comparative study in different cell types," Bioelectromagnetics, vol. 27, pp. 560-577, 2006. Available at: https://doi.org/10.1002/bem.20252.

[93] D. Wang, Z. Wang, L. Zhang, Z. Li, X. Tian, J. Fang, Q. Lu, and X. Zhang, "Cellular ATP levels are affected by moderate and strong static magnetic fields," Bioelectromagnetics, vol. 39, pp. 352-360, 2018. Available at: https://doi.org/10.1002/bem.22122.

[94] H. Jalilian, S. H. Teshnizi, M. Röösli, and M. Neghab, "Occupational exposure to extremely low frequency magnetic fields and risk of Alzheimer disease: A systematic review and meta-analysis," Neurotoxicology, vol. 69, pp. 242-252, 2017. Available at: https://doi.org/10.1016/j.neuro.2017.12.005.

[95] S. Bawin, L. Kaczmarek, and W. Adey, "Effects of modulated VHF fields on the central nervous system," Annals of the New York Academy of Sciences, vol. 247, pp. 74-81, 1975. Available at: https://doi.org/10.1111/j.1749-6632.1975.tb35984.x.

[96] S. M. Bawin and W. R. Adey, "Sensitivity of calcium binding in cerebral tissue to weak environmental electric fields oscillating at low frequency," Proceedings of the National Academy of Sciences, vol. 73, pp. 1999-2003, $1976 . \quad$ Available at: https://doi.org/10.1073/pnas.73.6.1999.

[97] C. Blackman, S. Benane, and D. House, "The influence of temperature during electric-and magnetic-field-induced alteration of calcium-ion C. Blackman, S. Benane, and D. House, "The influence of temperature during electric-and magnetic-field-induced alteration of calcium-ion
release from in vitro brain tissue," Bioelectromagnetics, vol. 12, pp. 173-182, 1991. Available at: https://doi.org/10.1002/bem.2250120305.

[98] M. Zmyslony, E. Rajkowska, P. Mamrot, P. Politanski, and J. Jajte, "The effect of weak $50 \mathrm{~Hz}$ magnetic fields on the number of free oxygen radicals in rat lymphocytes in vitro," Bioelectromagnetics, vol. 25, pp. 607-612, 2004. Available at: https://doi.org/10.1002/bem.20045.

[99] T. Köhler, M. Wölfel, M. Ciba, U. Bochtler, and C. Thielemann, "Terrestrial trunked radio (TETRA) exposure of neuronal in vitro networks," Environmental Research, vol. 162, pp. 1-7, 2018. Available at: https://doi.org/10.1016/j.envres.2017.12.007.

[100] E. Valentini, G. Curcio, F. Moroni, M. Ferrara, L. De Gennaro, and M. Bertini, "Neurophysiological effects of mobile phone electromagnetic fields on humans: A comprehensive review," Bioelectromagnetics, vol. 28, pp. 415-432, 2007. Available at: https://doi.org/10.1002/bem.20323.

[101] F. Apollonio, M. Liberti, A. Paffi, C. Merla, P. Marracino, A. Denzi, C. Marino, and G. d'Inzeo, "Feasibility for microwaves energy to affect biological systems via nonthermal mechanisms: A systematic approach," IEEE Transactions on Microwave Theory and Techniques, vol. 61, pp. 2031-2045, 2013. Available at: https://doi.org/10.1 109/tmtt.2013.2250298.

[102] M. Simkó, D. Remondini, O. Zeni, and M. R. Scarfi, "Quality matters: Systematic analysis of endpoints related to cellular life in Vitro data of radiofrequency electromagnetic field exposure," International Journal of Environmental Research and Public Health, vol. 13, pp. 701-701, 2016. Available at: https://doi.org/10.3390/ijerph13070701.

[103] R. Ding, Y. J. Zhao, F. L. Chen, J. Z. Chen, and S. X. Duan, "Magnetization mechanism of magnetized water," Acta Physica Sinica, vol. 60, p. $064701,2011$. 